\title{
Nomogram based on clinical characteristics and serological inflammation markers to predict overall survival of oral tongue squamous cell carcinoma patient after surgery
}

Yi-Wei Lin ${ }^{1,2+}$, Wei-Piao Kang ${ }^{3 \dagger}$, Bin-Liang Huang ${ }^{1,2}$, Zi-Han Qiu ${ }^{6}$, Lai-Feng Wei ${ }^{1,2}$, Biao Zhang ${ }^{1,2}$, Tian-Yan Ding ${ }^{1,2}$, Yun Luo ${ }^{1,2}$, Can-Tong Liu' ${ }^{1,2}$, Ling-Yu Chu ${ }^{1,2}$, Hai-Peng Guo ${ }^{4^{*}}$, Yi-Wei Xu ${ }^{1,2,5^{*}}$ and Yu-Hui Peng ${ }^{1,2,5^{*}}$

\begin{abstract}
Background: Oral tongue squamous cell carcinoma (OTSCC) is a prevalent malignant disease that is characterized by high rates of metastasis and postoperative recurrence. The aim of this study was to establish a nomogram to predict the outcome of OTSCC patients after surgery.

Methods: We retrospectively analyzed 169 OTSCC patients who underwent treatments in the Cancer Hospital of Shantou University Medical College from 2008 to 2019. The Cox regression analysis was performed to determine the independent prognostic factors associated with patient's overall survival (OS). A nomogram based on these prognostic factors was established and internally validated using a bootstrap resampling method.

Results: Multivariate Cox regression analysis revealed the independent prognostic factors for OS were TNM stage, age, lymphocyte-to-monocyte ratio and immunoglobulin $\mathrm{G}$, all of which were identified to create the nomogram. The Akaike Information Criterion and Bayesian Information Criterion of the nomogram were lower than those of TNM stage (292.222 vs. 305.480; 298.444 vs. 307.036, respectively), indicating a better goodness-of-fit of the nomogram for predicting OS. The bootstrap-corrected of concordance index (C-index) of nomogram was 0.784 (95\% Cl 0.708-0.860), which was higher than that of TNM stage $(0.685,95 \% \mathrm{Cl} 0.603-0.767, P=0.017)$. The results of time-dependent $\mathrm{C}$-index for OS also showed that the nomogram had a better discriminative ability than that of TNM stage. The calibration curves of the nomogram showed good consistency between the probabilities and observed values. The decision curve analysis also revealed the potential clinical usefulness of the nomogram. Based on the cutoff value obtained from the nomogram, the proposed high-risk group had poorer OS than low-risk group $(P<0.0001)$.
\end{abstract}

\footnotetext{
*Correspondence: ghaipeng@sina.cn; yiwei512@126.com;

pengyuhui666@163.com

${ }^{\dagger} Y$ i-Wei Lin and Wei-Piao Kang contributed equally to this work

1 Department of Clinical Laboratory Medicine, The Cancer Hospital of Shantou University Medical College, Shantou 515041, Guangdong, People's Republic of China

${ }^{4}$ Department of Head and Neck Surgery, The Cancer Hospital of Shantou

University Medical College, Shantou 515041, Guangdong, People's

Republic of China

Full list of author information is available at the end of the article
}

(C) The Author(s) 2021. Open Access This article is licensed under a Creative Commons Attribution 4.0 International License, which permits use, sharing, adaptation, distribution and reproduction in any medium or format, as long as you give appropriate credit to the original author(s) and the source, provide a link to the Creative Commons licence, and indicate if changes were made. The images or other third party material in this article are included in the article's Creative Commons licence, unless indicated otherwise in a credit line to the material. If material is not included in the article's Creative Commons licence and your intended use is not permitted by statutory regulation or exceeds the permitted use, you will need to obtain permission directly from the copyright holder. To view a copy of this licence, visit http://creativecommons.org/licenses/by/4.0/. The Creative Commons Public Domain Dedication waiver (http://creativeco mmons.org/publicdomain/zero/1.0/) applies to the data made available in this article, unless otherwise stated in a credit line to the data. 
Conclusions: The nomogram based on clinical characteristics and serological inflammation markers might be useful for outcome prediction of OTSCC patient.

Keywords: Oral tongue squamous cell carcinoma, Inflammation markers, Pretreatment nomogram, Prognosis, Surgery

\section{Introduction}

Oral tongue squamous cell carcinoma (OTSCC) is one of the most prevalent malignancy of the oral cavity worldwide with a relatively poor prognosis [1]. High rates of local recurrence and cervical lymph node metastasis are the most notorious clinical behaviors of OTSCC, which usually cause impairment of patient's speech, mastication and deglutition $[2,3]$. The 5-year survival rate of OTSCC patients is still unsatisfactory even with combined treatments involving surgery, radiotherapy and chemotherapy $[4,5]$. Owing to the diverse clinical pathological characteristics of patients, it is important to predict the outcome of OTSCC patients for the selection of more personalized treatment strategies.

At present, the TNM staging system is the gold standard for prognostication in oncology, but it still has limitations. One of the primary disadvantages is its inability to incorporate other variables, such as genetic differences and patient characteristics including age, gender and race, to predict prognosis of cancer patients [6]. Moreover, identifying clinically significant and inexpensiveto-measure prognostic factors obtained before surgery would provide more valuable insights to help clinicians and OTSCC patients to choose appropriate treatment strategy. Hence, it is necessary to identify a robust prognostic tool that can integrate these potential prognostic factors to complement the TNM staging system to better predict the outcome of OTSCC patients. Nomogram is a reliable, user-friendly and sophisticated statistical prediction tool, with the ability to estimate individualized risk via incorporating variables based on the patient and disease characteristics [7]. Nomograms have been widely used for estimating recurrence $[8,9]$, specific survival $[10,11]$, overall survival $[12,13]$ of tumor patients, and may assist clinicians in making individual treatment strategies [6].

Systemic inflammation has been reported to play an important role in the pathogenesis and progression of cancer [14]. Moreover, the association between serological inflammation markers and prognosis of human malignancies has been reported. For example, higher pre-treatment lymphocyte-to-monocyte ratio (LMR) have been shown to be associated with a better prognosis in various tumors [15-18]. And the lower immunoglobulin G (IgG) levels predicted an elevated risk of developing pancreatic cancer compared to the reference levels [19]. Thus, the current study aimed to establish a nomogram to predict OTSCC patient's outcome based on clinical characteristics and serological inflammation markers which are easy to obtain from routine admission laboratory tests, and assessed the performance of the nomogram with internal validation using a bootstrap resampling method.

\section{Materials and methods \\ Study population}

This retrospective study consisted of 169 patients with pathologically-proven OTSCC in the Cancer Hospital of Shantou University Medical College between July 2008 and February 2019 (Fig. 1). Patients included in this study met the following criteria: (1) Tumors were histologically confirmed to be OTSCC. (2) All patients received primary surgical resection but had not undergone preoperative cancer-related treatment. (3) Patients who suffered from any cancers or autoimmune diseases before OTSCC diagnosis were excluded from this study. (4) All patients had complete baseline clinical information and follow-up data. The median follow-up time of patients was 50 months, and the minimum and maximum followup time was 1 month and 130 months, respectively. The overall survival (OS) was defined as the interval from the initial diagnosis to either any form of death or the last follow-up time. The last follow-up was performed in

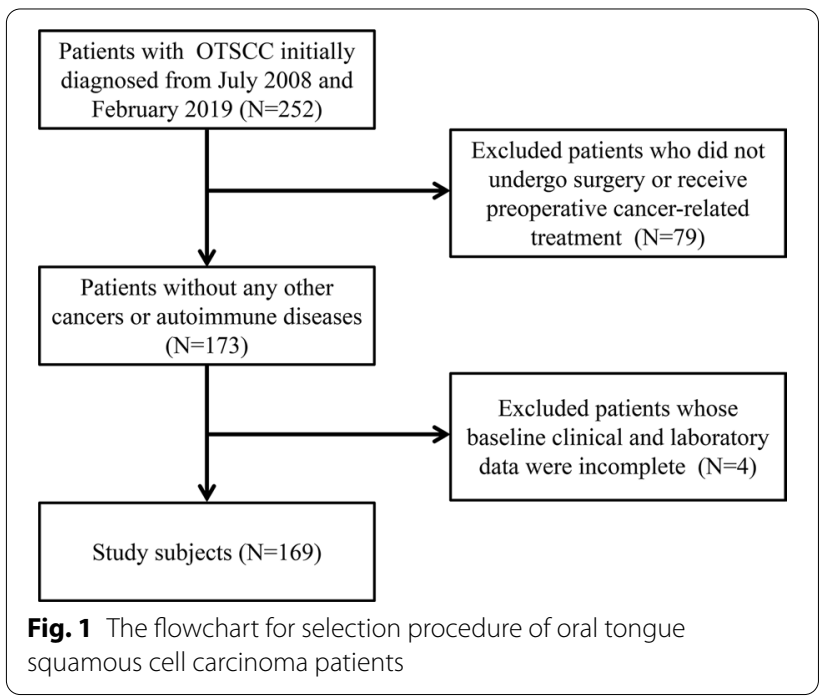


September 2019. This study was approved by the Hospital Ethics Committee in Cancer Hospital of Shantou University Medical College and informed consents were obtained from all included participants. All work was complied with the principles of the Helsinki Declaration.

Clinical baseline data of each patient was collected as follows: clinical pathological TNM stage, gender, age and tumor size. Tumor stage were classified according to the eighth edition of the Union for International Cancer Control/American Joint Cancer Committee (AJCC) TNM staging system [20]. Peripheral blood samples of the patients were collected before surgery began. The potential serum prognostic factors included IgG, immunoglobulin A (IgA), immunoglobulin M (IgM), Complement 3 (C3), Complement 4 (C4), B factor (BF), C-reactive protein (CRP), white blood cell count (WBC), platelet-tolymphocyte ratio (PLR), neutrophil-to-lymphocyte ratio (NLR) and LMR.

\section{Model construction and assessment}

In this study, continuous variables were transformed into categorical variables and the optimal cut-off values for the continuous variables were obtained by X-tile [21]. Prognostic factors for OS were selected by Cox proportional hazards regression analysis, and those with a significant level of $P \leq 0.10$ in univariate analysis were analyzed using multivariate Cox regression analysis. A nomogram with endpoints of 1-, 3- and 5-year OS was constructed based on the multivariate analysis results. By comparing with selected prognostic factors and TNM stage, the goodness-of-fit and discriminative ability of the nomogram were examined with Akaike Information Criterion (AIC) and Bayesian Information Criterion (BIC), and concordance index (C-index), respectively. The calibration of the nomogram was assessed with calibration curve, and decision curve analysis was conducted to estimate the clinical utility of the nomogram. The Kaplan-Meier method and log-rank test were applied for calculating and comparing the differences in OS. All internal validations were performed using bootstrapping method with 1,000 resamples.

\section{Statistical analyses}

Statistical analyses were performed using SPSS software, version 19.0 (SPSS Inc., Chicago, IL, USA) and R (version 4.0.2) for Windows. The nomogram, decision curve analysis curves and calibration curves were plotted by the rms package in $\mathrm{R}$. Time-dependent $\mathrm{C}$-index curves were plotted by the pec package in R. Survival curve was plotted using Kaplan-Meier survival analysis and compared using the log-rank test with the survminer and survival package in R. $P<0.05$ was considered statistically significant.

\section{Results}

\section{Patient characteristics}

We recruited 169 OOTSCC patients with complete baseline clinical and laboratory data (Fig. 1). The clinical characteristics of these patients were shown in Table 1. The median age for patients was 57 years (range 25-88 years), of which 93 (55\%) were males and 76 (45\%) were females. The numbers of patient with I-II and IIIIV stage were $147(87 \%)$ and $22(13 \%)$, respectively. The optimal cut-off values for the continuous variables were obtained by X-tile as follows: age $(69 \mathrm{y}), \operatorname{IgG}(12.51 \mathrm{~g} / \mathrm{L}$, range 6.06-49.02), IgA (1.97 g/L, range 0.48-4.58), IgM (1.09 g/L, range 0.32-3.61), C3 (1.05 g/L, range $0.42-$ 2.05), C4 (0.22 g/L, range 0.097-0.516), BF $(0.47 \mathrm{~g} / \mathrm{L}$, range $0.2-0.92)$, CRP $(3.78 \mathrm{mg} / \mathrm{L}$, range $0.55-69.39)$, WBC $\left(4.95 \times 10^{9} / \mathrm{L}\right.$, range $\left.3.1-12.2\right)$, LMR (4.15, range 1.36-10.2), NLR (1.61, range 0.44-7.7) and PLR (177.86, range $36.98-548.86)$.

\section{Construction of the nomogram based on clinical and serological markers}

The univariate and multivariate Cox analysis were used to select the potential prognostic markers, and estimate their influence on OS for OTSCC patients. The multivariate analysis results showed that the following variables remained significantly independent prognostic factors: TNM stage $(P<0.001, \mathrm{HR}=4.712 ; 95 \%$ CI $2.272-9.770)$, age $(P=0.008, \mathrm{HR}=3.253 ; 95 \%$ CI $1.353-7.825)$, LMR $(P=0.035, \mathrm{HR}=0.408 ; 95 \% \mathrm{CI} 0.177-0.940)$ and IgG $(P=0.017, \mathrm{HR}=0.385 ; 95 \% \mathrm{CI} 0.176-0.840)$ (Fig. 2). The detailed results of univariate and multivariate analyses were presented in Table 2.

Incorporating these prognostic markers including TNM stage, age, LMR and IgG, the nomogram was constructed for 1-, 3- and 5-year OS prediction (Fig. 3). From the nomogram, each prognostic factor had a risk point, which could be obtained by drawing a vertical line directly upward from the corresponding value of the prognostic factor to an axis with "Points". In order to determine the 1-, 3-, and 5-year OS probability of a specific patient, a vertical line could be drawn from the "Total Points" which was the sum of the risk points of all prognostic factors, to the axis marked "1-, 3-, and 5-year OS". And a larger "Total Points" score would represented a worse OS for the patient.

\section{The goodness-of-fit and discrimination of the nomogram}

The goodness-of-fit and discriminative ability of the nomogram were examined by the AIC and BIC, and 
Table 1 Demographics and clinical characteristics of OTSCC patients

\begin{tabular}{|c|c|c|}
\hline Characteristics & $\begin{array}{l}\text { No. of Patients } \\
(\mathrm{N}=169), \mathrm{n}(\%)\end{array}$ & $\begin{array}{l}\text { No. of Events } \\
(\mathrm{N}=35), \mathrm{n}(\%)\end{array}$ \\
\hline \multicolumn{3}{|l|}{ Gender } \\
\hline Male & $93(55)$ & $19(54)$ \\
\hline Female & $76(45)$ & $16(46)$ \\
\hline \multicolumn{3}{|l|}{ Age (years) } \\
\hline$<69$ & $141(84)$ & $27(77)$ \\
\hline$\geq 69$ & $28(16)$ & $8(23)$ \\
\hline \multicolumn{3}{|l|}{ Tumor size (cm) } \\
\hline$<4.2$ & $150(89)$ & $26(74)$ \\
\hline$\geq 4.2$ & $19(11)$ & $9(26)$ \\
\hline \multicolumn{3}{|l|}{ TNM stage } \\
\hline$|-| \mid$ & $147(87)$ & $14(40)$ \\
\hline III-IV & $22(13)$ & $21(60)$ \\
\hline \multicolumn{3}{|l|}{ Treatment } \\
\hline Surgery & $121(72)$ & $14(40)$ \\
\hline $\begin{array}{l}\text { Surgery followed by radio- } \\
\text { therapy/chemotherapy }\end{array}$ & $48(28)$ & $21(60)$ \\
\hline \multicolumn{3}{|l|}{$\operatorname{lgG}(g / L)$} \\
\hline$<12.51$ & $85(50)$ & $23(66)$ \\
\hline$\geq 12.51$ & $84(50)$ & $12(34)$ \\
\hline \multicolumn{3}{|l|}{$\lg A(g / L)$} \\
\hline$<1.97$ & $73(43)$ & $15(43)$ \\
\hline$\geq 1.97$ & $96(57)$ & $20(57)$ \\
\hline \multicolumn{3}{|l|}{$\lg M(g / L)$} \\
\hline$<1.09$ & $78(46)$ & $19(54)$ \\
\hline$\geq 1.09$ & $91(54)$ & $16(46)$ \\
\hline \multicolumn{3}{|l|}{ C3 (g/L) } \\
\hline$<1.05$ & $86(51)$ & $15(43)$ \\
\hline$\geq 1.05$ & $83(49)$ & $20(57)$ \\
\hline \multicolumn{3}{|l|}{$\mathrm{C} 4(\mathrm{~g} / \mathrm{L})$} \\
\hline$<0.22$ & $56(33)$ & $11(31)$ \\
\hline$\geq 0.22$ & $113(67)$ & $24(69)$ \\
\hline \multicolumn{3}{|l|}{ BF (g/L) } \\
\hline$<0.47$ & $142(85)$ & $29(83)$ \\
\hline$\geq 0.47$ & $27(15)$ & $6(17)$ \\
\hline \multicolumn{3}{|l|}{$\mathrm{CRP}(\mathrm{mg} / \mathrm{L})$} \\
\hline$<3.78$ & $143(85)$ & $28(80)$ \\
\hline$\geq 3.78$ & $26(15)$ & $7(20)$ \\
\hline \multicolumn{3}{|l|}{ WBC $\left(\times 10^{9} / \mathrm{L}\right)$} \\
\hline$<4.95$ & $30(17)$ & $9(26)$ \\
\hline$\geq 4.95$ & $139(83)$ & $26(74)$ \\
\hline \multicolumn{3}{|l|}{ PLR } \\
\hline$<177.86$ & $148(88)$ & $29(83)$ \\
\hline$\geq 177.86$ & $21(12)$ & $6(17)$ \\
\hline \multicolumn{3}{|l|}{ NLR } \\
\hline$<1.61$ & $63(37)$ & $16(46)$ \\
\hline$\geq 1.61$ & $106(63)$ & $19(54)$ \\
\hline \multicolumn{3}{|l|}{ LMR } \\
\hline$<4.15$ & $96(57)$ & $27(77)$ \\
\hline$\geq 4.16$ & $73(43)$ & $8(23)$ \\
\hline
\end{tabular}

Table 1 (continued)

TNM, tumor/node/metastasis; OTSCC, oral tongue squamous cell carcinoma; IgG, immunoglobulin $\mathrm{G}$; IgA, immunoglobulin $\mathrm{A}$; IgM, immunoglobulin $\mathrm{M} ; \mathrm{C} 3$, Complement 3; C4, Complement 4; BF, B factor; CRP, C-reactive protein; WBC, white blood cell count; PLR, platelet-to-lymphocyte ratio; NLR, neutrophil-tolymphocyte ratio; LMR, lymphocyte-to-monocyte ratio

C-index, respectively. The results were presented in Table 3. The AIC and BIC of the nomogram for OS were much lower than those of TNM stage (292.222 vs. $305.480 ; 298.444$ vs. 307.036 , respectively), indicating that the nomogram had a higher goodness-of-fit for predicting OS. The bootstrap-corrected C-index of the nomogram was 0.784 (95\% CI 0.708-0.860), which was higher than that of TNM stage $(0.685,95 \%$ CI $0.603-0.767$, $P=0.017)$. Moreover, time-dependent $\mathrm{C}$-index analysis also showed that the nomogram model exhibited good prognostic accuracy in clinical outcome prediction either for 1-, 3- and 5-year OS of patient when compared with TNM stage and any single prognostic marker (Fig. 4A). A similar result was also observed in internally validation using a bootstrap resampling method (Fig. 4B).

\section{Net benefit and predictive capacity of the nomogram}

The decision curve analysis and calibration curves were used to determine net benefit and predictive capacity of the nomogram. As shown in Fig. 5, the decision curves analysis for 1-, 3-, and 5-year OS showed that the nomogram had higher overall net benefit compared with traditional TNM stage across the majority of the range of reasonable threshold probabilities. In addition, calibration would estimate how close the nomogram estimated risk was to the observed risk, depicted by a calibration plot. Figure 6 illustrated the good calibration of our nomogram for the 1-, 3-, and 5-year OS predictions. Taken together, these results demonstrated that our nomogram had a better performance to predict survival outcomes of OTSCC patients when compared with TNM stage.

\section{Risk stratification based on the nomogram}

To assess whether the OTSCC patients could be effectively separated into two proposed risk groups based on the nomogram and OS, we calculated each patient's total point and used the X-tile program to determine the optimal cutoff value. Using the cutoff value of 195.13, the OTSCC patients were subdivided into low- and highrisk groups, and Kaplan-Meier survival analysis was applied to assess their survival. Compared with patients in the low-risk group whose median OS was 47 months, patients in the high-risk group had shorter OS (median OS: 15 months; $P<0.0001$; Fig. 7 ). 


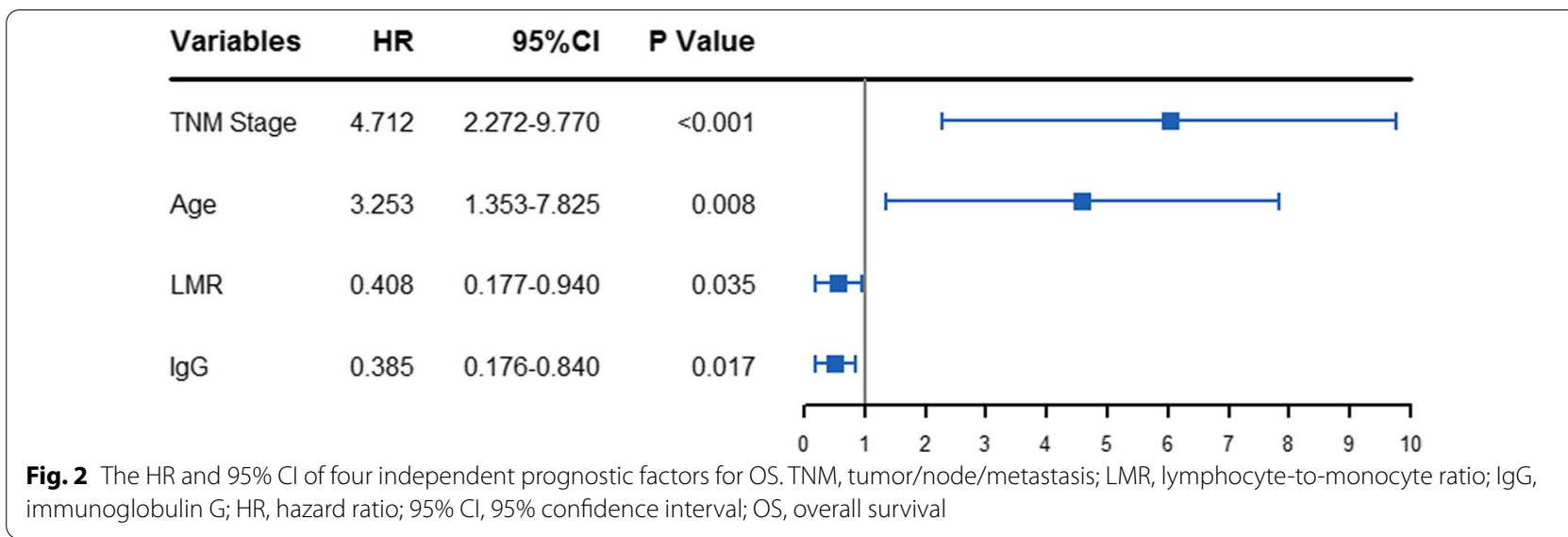

Table 2 Univariate and multivariate Cox proportional hazards regression analysis for OS

\begin{tabular}{|c|c|c|c|c|c|c|}
\hline & \multicolumn{3}{|c|}{ Univariate analysis } & \multicolumn{3}{|c|}{ Multivariate analysis } \\
\hline & HR & $95 \% \mathrm{Cl}$ & $P$ & HR & $95 \% \mathrm{Cl}$ & $P$ \\
\hline Gender (female vs. male) & 1.099 & $0.565-2.137$ & 0.782 & & & \\
\hline Age ( $\geq 69$ vs. $<69 ; y)$ & 1.997 & $0.905-4.408$ & 0.087 & 3.253 & $1.353-7.825$ & 0.008 \\
\hline Tumor size ( $\geq 4.2$ vs. $<4.2 ; \mathrm{cm})$ & 2.945 & $1.376-6.304$ & 0.005 & & & \\
\hline TNM Stage (III-IV vs. I-II) & 4.396 & $2.230-8.664$ & 0.000 & 4.712 & $2.272-9.770$ & 0.000 \\
\hline $\operatorname{lgG}(\geq 12.51$ vs. $<12.51 ; \mathrm{g} / \mathrm{L})$ & 0.457 & $0.225-0.926$ & 0.030 & 0.385 & $0.176-0.840$ & 0.017 \\
\hline $\lg \mathrm{A}(\geq 1.97$ vs. $<1.97 ; \mathrm{g} / \mathrm{L})$ & 1.474 & $0.773-2.812$ & 0.239 & & & \\
\hline $\operatorname{lgM}(\geq 1.09$ vs. < 1.09; g/L) & 0.538 & $0.273-1.060$ & 0.073 & & & \\
\hline C3 ( $\geq 1.05$ vs. $<1.05 ; g / L)$ & 1.924 & $0.967-3.830$ & 0.062 & & & \\
\hline$C 4(\geq 0.22$ vs. $<0.22 ; g / L)$ & 1.582 & $0.773-3.235$ & 0.209 & & & \\
\hline $\mathrm{BF}(\geq 0.47$ vs. $<0.47 ; \mathrm{g} / \mathrm{L})$ & 2.087 & $0.876-4.968$ & 0.097 & & & \\
\hline CRP ( $\geq 3.78$ vs. $<3.78 ; \mathrm{mg} / \mathrm{L})$ & 1.621 & $0.722-3.638$ & 0.241 & & & \\
\hline WBC $\left(\geq 4.95\right.$ vs. $\left.<4.95 ; 10^{9} / L\right)$ & 0.467 & $0.218-1.003$ & 0.051 & & & \\
\hline $\operatorname{LMR}(\geq 4.15$ vs. $<4.15)$ & 0.243 & $0.110-0.539$ & 0.001 & 0.408 & $0.177-0.940$ & 0.035 \\
\hline $\operatorname{PLR}(\geq 177.86$ vs. $<177.86)$ & 2.205 & $0.913-5.325$ & 0.079 & & & \\
\hline $\operatorname{NLR}(\geq 1.61$ vs. $<1.61)$ & 0.682 & $0.351-1.328$ & 0.261 & & & \\
\hline
\end{tabular}

HR, Hazard ratio; 95\% Cl, 95\% confidence interval; TNM, tumor/node/metastasis; lgG, immunoglobulin G; IgA, immunoglobulin A; lgM, immunoglobulin M; C3, Complement 3; C4, Complement 4; BF, B factor; CRP, C-reactive protein; WBC, white blood cell count; LMR, lymphocyte-to-monocyte ratio; PLR, platelet-to-lymphocyte ratio; NLR, neutrophil-to-lymphocyte ratio; OS, overall survival

\section{Discussion}

OTSCC is one of the most aggressive tumors of oral cavity with a relatively poor prognosis [1]. At present, the prognosis and treatment of OTSCC patients are primarily determined by the AJCC TNM staging system. However, even at the same stage of OTSCC, the prognosis of patients is still highly different since it is influenced by a variety of factors [22]. Therefore, in order to find other reliable prognostic factors to complement the TNM stage system to better predict patient's outcomes and help guide treatments, we used Cox proportional hazards regression analysis to determine TNM stage, age, LMR, IgG as independent prognostic factors for OS.
Then, a nomogram that incorporated these four prognostic factors to predict OS of OTSCC patients was established and internally validated. Our nomogram showed enhanced predictive accuracy and discriminative ability when compared with the traditional TNM stage system. Moreover, the nomogram signature successfully separated OTSCC patients into high-risk and low-risk groups with significant differences in OS.

To date, increasing research have indicated a significant link between systemic inflammatory response and progression and prognosis of various types of tumors [23]. It has shown that several pretreatment peripheral indicators of immunity/inflammation are significant factors in 


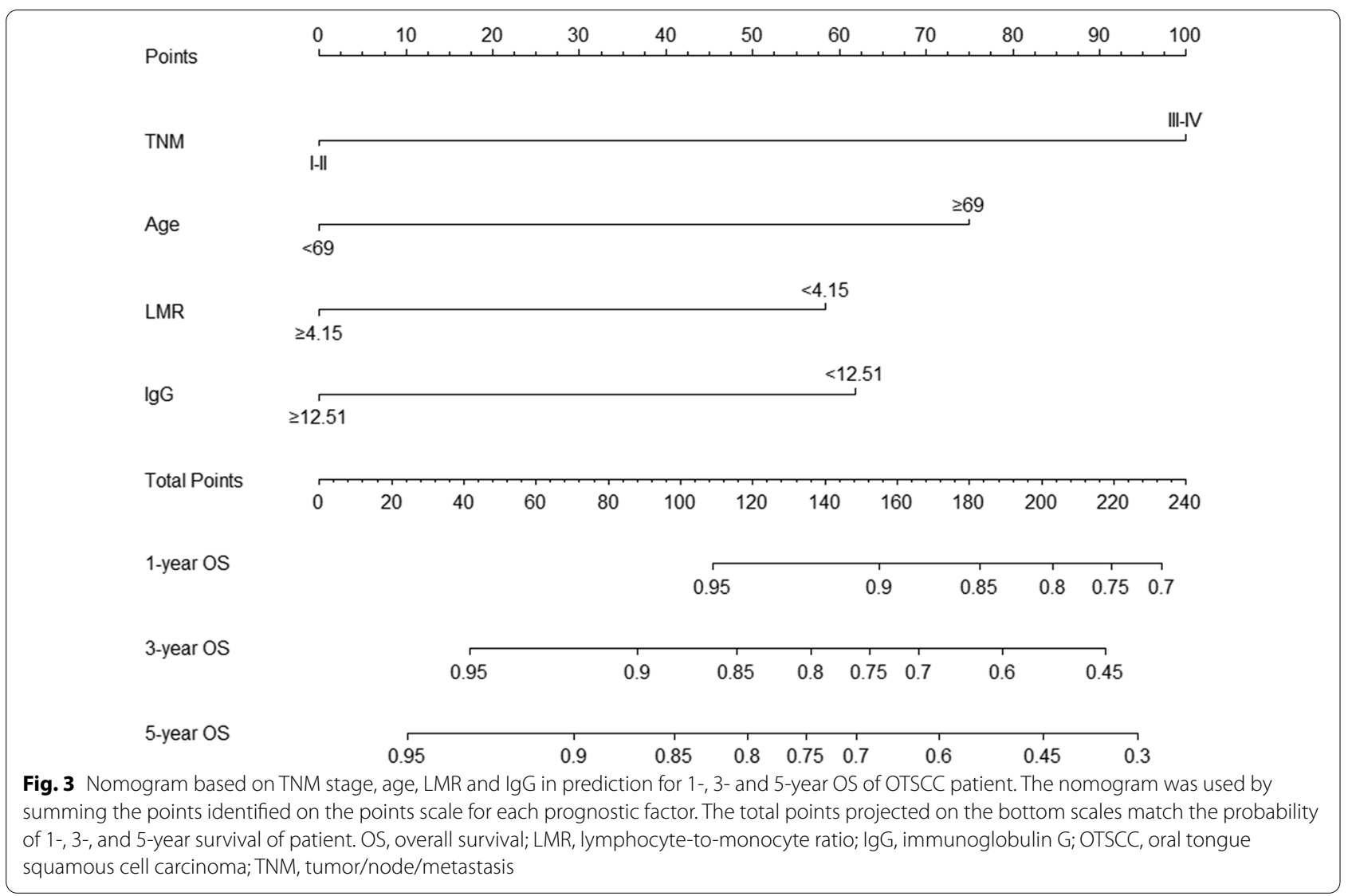

Table 3 The AIC, BIC and C-index of prognostic factors and nomogram for prediction OS

\begin{tabular}{|c|c|c|c|c|}
\hline & C-index $(95 \% \mathrm{Cl})$ & $P$-value & AIC & $\mathrm{BIC}$ \\
\hline TNM & $0.685(0.603-0.767)$ & & 305.480 & 307.036 \\
\hline Age & $0.556(0.480-0.632)$ & & 321.534 & 323.089 \\
\hline LMR & $0.638(0.558-0.718)$ & & 309.457 & 311.012 \\
\hline $\lg G$ & $0.602(0.518-0.686)$ & & 318.899 & 320.454 \\
\hline Nomogram & $0.784(0.708-0.860)$ & & 292.222 & 298.444 \\
\hline $\begin{array}{l}\text { Nomogram versus } \\
\text { TNM }\end{array}$ & & 0.017 & & \\
\hline $\begin{array}{l}\text { Nomogram versus } \\
\text { Age }\end{array}$ & & $<0.001$ & & \\
\hline $\begin{array}{l}\text { Nomogram versus } \\
\text { LMR }\end{array}$ & & $<0.001$ & & \\
\hline $\begin{array}{l}\text { Nomogram versus } \\
\text { lgG }\end{array}$ & & $<0.001$ & & \\
\hline
\end{tabular}

C-index, concordance index; $95 \% \mathrm{Cl}, 95 \%$ confidence interval; AIC, Akaike Information Criterion; BIC, Bayesian Information Criterion; LMR, lymphocyteto-monocyte ratio; IgG, immunoglobulin G; TNM, tumor/node/metastasis; OS, overall survival; $P$-values are calculated based on normal approximation using function rcorrp.cens in Hmisc package predicting the progression and prognosis of tumors $[16$, 24]. LMR, as one of the immunity/inflammation indicators, was studied for possible correlation with OTSCC patient's outcome in this study. LMR has been reported to enter into prognostic nomograms for pancreatic [16], colorectal [17], epithelial [18], tongue [25], and other solid cancers [15]. All these studies suggested that a higher LMR was related to favorable prognosis, which was also confirmed in our study $(\mathrm{HR}=0.408 ; 95 \% \mathrm{CI}$ $0.177-0.940 ; P=0.035)$. Although the prognostic value of LMR in tumors seems clear, the actual mechanisms by which it contributes to improve survival outcome require further study. LMR is defined as the absolute peripheral lymphocyte count divided by the absolute peripheral monocyte count. Admittedly, lymphocytes play a critical role in the immune response and destroy residual cancer cells by recognizing tumor antigens [23]. Moreover, Tumor-infiltrating lymphocytes (TILs) are thought to be responsible for cellular as well as humoral antitumor immune responses that contribute to suppress tumor proliferation, invasion, and metastasis. Indeed, higher numbers of TILs were associated with better clinical outcomes [26, 27], and lymphopenia was found to be correlated to worse OS in prospectively collected series 

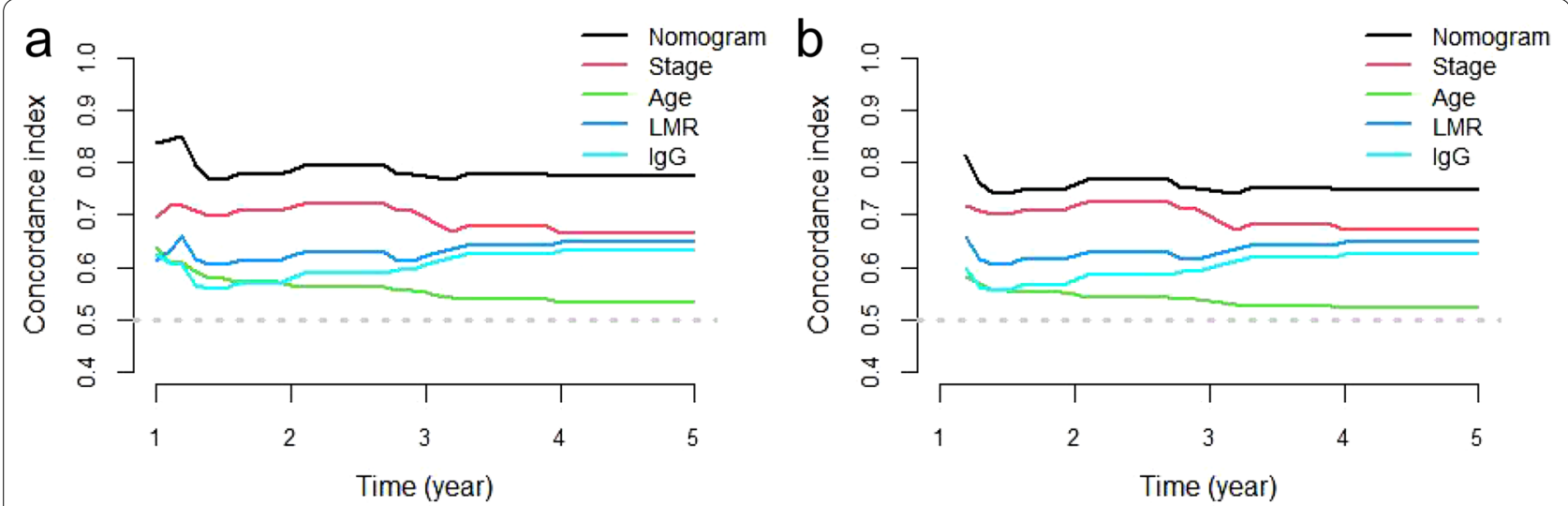

Fig. 4 Time-dependent C-index of nomogram compared with TNM stage, age, LMR and IgG for OS of OTSCC patient (a) and internally validated with using a bootstrap resampling method (b). C-index, concordance index; OS, overall survival; LMR, lymphocyte-to-monocyte ratio; lgG, immunoglobulin G; OTSCC, oral tongue squamous cell carcinoma; TNM, tumor/node/metastasis
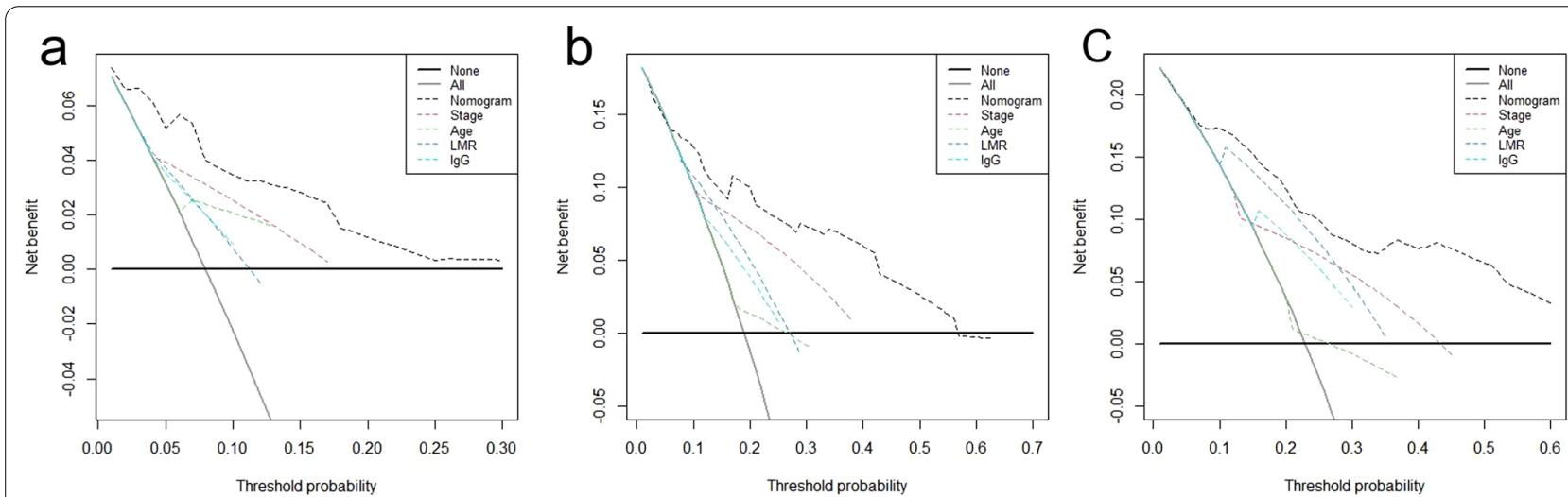

Fig. 5 Decision curve analysis of nomogram compared with TNM stage, age, LMR and lgG for 1-year OS (a), 3-year OS (b), 5-year OS (c) of OTSCC patient. The thick grey line is the net benefit for a strategy of treating all men; the thick black line is the net benefit of treating no men. The $y$-axis indicates the overall net benefit, which is calculated by summing the benefits (true positive results) and subtracting the harms (false positive results). OS, overall survival; LMR, lymphocyte-to-monocyte ratio; lgG, immunoglobulin G; OTSCC, oral tongue squamous cell carcinoma; TNM, tumor/node/metastasis

of patients with metastatic breast cancer, non-Hodgkin lymphoma, and soft tissue sarcoma [28]. On the other hand, a higher monocyte count has been presented as a poor prognostic factor in metastatic melanoma [29] and cervical cancer [30]. Tumor-associated macrophages, which are derived from circulating monocytes and accelerate tumor progression by production of growth factors and cytokines, have also been suggested to be related to unfavorable prognosis in breast cancer [31], and Hodgkin's lymphoma [32]. Thus, a lower LMR may indicate an imbalance of the inflammatory response, which would be reflected by a weak antitumor immunity and favorable microenvironment for tumor growth.
IgG represents a highly abundant antibody subtype in human serum and is a key component in anti-tumor humoral immune response [33]. A number of studies have demonstrated that aberrant post-translational modifications of IgG are responsible for numerous pathological processes including cancer [34-36]. Moreover, the evidence for an inverse association between pre-diagnostic serum IgG level and the risk of developing melanoma or pancreatic cancer was found in the Swedish Apolipoprotein-related MORtality RISk (AMORIS) cohort study. The humoral response might provide a protective role against the development of melanoma or pancreatic cancer, mediated through IgG $[19,37]$. In the current study, we revealed that a higher 


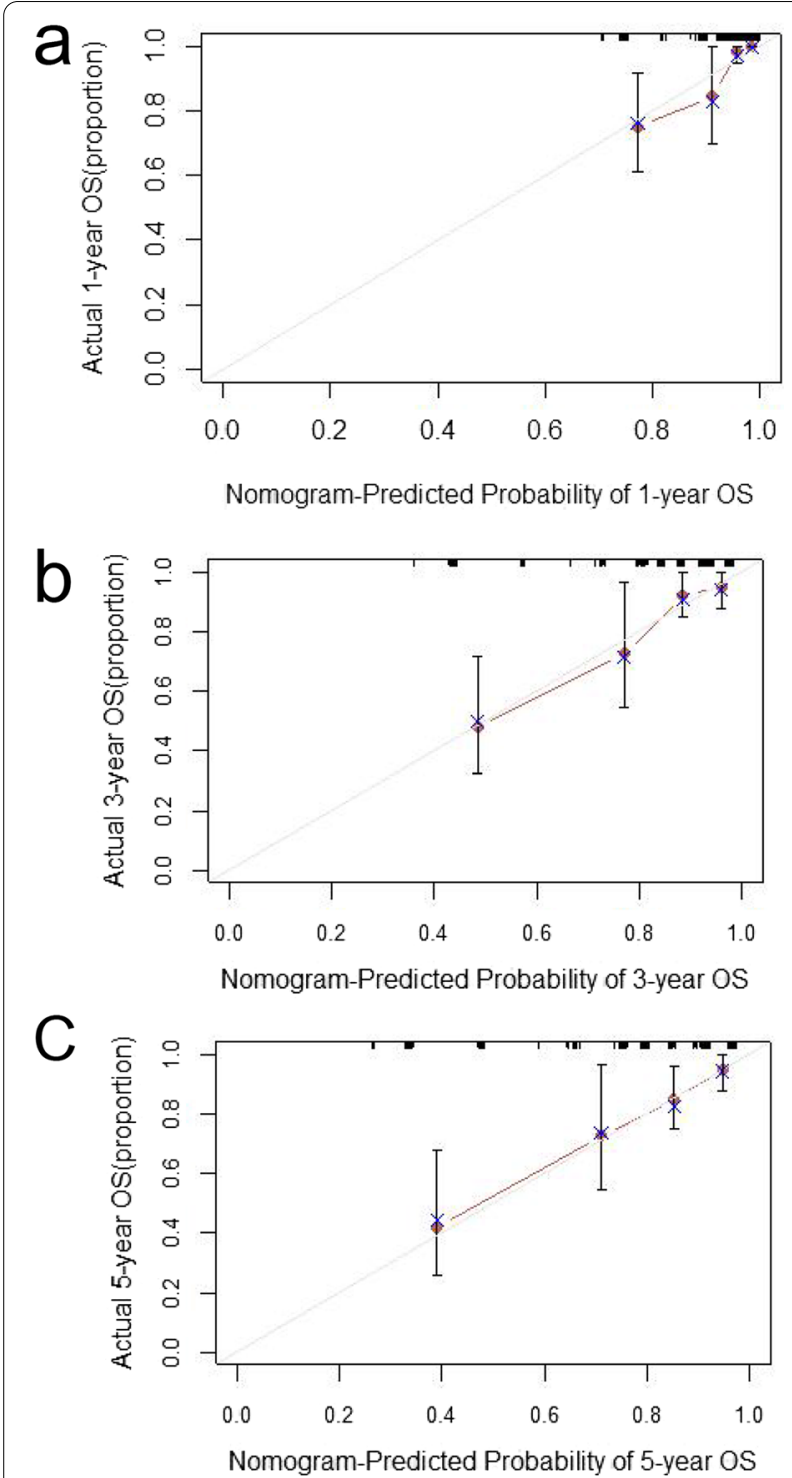

Fig. 6 Calibration curves for 1-year OS (a), 3-year OS (b), 5-year OS (c) of nomogram predictions. OS, overall survival

IgG level was not only an independent prognostic factor, but was also associated with better OS in OTSCC patients $(\mathrm{HR}=0.385 ; 95 \% \mathrm{CI} 0.176-0.840 ; P=0.017)$. This association may be explained by humoral response, which would play a critical role in suppression of tumor behaviors.

Recently, a wide variety of prognostic nomograms based on patients' demographics and clinicopathological parameters, such as age, gender, race, tumor site and depth of tumor invasion, have been developed for survival prediction of patients with tongue cancer [2, 38-45]. On the other hand, most studies indicated that single serological inflammation marker, such as CRP [46],
NLR $[47,48]$ and LMR [25], could serve as an independent prognostic factor for OTSCC patients' survival prediction. Together, these findings might help clinicians to identify patients that would benefit from surgical resection and/or neck dissection strategies or, alternatively, if additional treatment methods need to be explored. In order to comprehensively improve prognostic accuracy and develop a multi-parametric prognostic model, number of potential serological inflammation markers and patients' demographics and clinicopathological features were included and assessed together in the current study. The results showed that our nomogram based on TNM stage, age, LMR and IgG, had incremental prognostic value compared to the traditional TNM staging system or any single serological inflammation marker. A similar study by Lu et al. also established a nomogram incorporating patients' clinicopathological factors and serological inflammation marker to predict survival for patients with OTSCC [22]. However, the serological inflammation markers included in Lu et al.s study were LMR, NLR, PLR and SII(systemic immune-inflammation index), and their nomogram construction was based on age, lymph node density (LND) and SII, which were different from ours. In our study, we collected more serological inflammation markers to evaluate the prognostic value for predicting OTSCC patients' survival, such as IgG, IgA, IgM, $\mathrm{C} 3, \mathrm{C} 4$ and $\mathrm{BF}$, all of which played an important role in development and progression of cancer, and might have potential prognostic value for cancer patient's survival prediction [49-56]. Moreover, we identified IgG could served as an independent prognostic factor for survival prediction of OTSCC patients, and higher IgG level was associated with better OS in OTSCC patients.

Taken together, based on the results of Cox regression analysis, our nomogram consisted of four prognostic factors: TNM stage, age, LMR and IgG. The data showed that our nomogram had a better goodness-of-fit for predicting OS of OTSCC patient. And the C-index of the nomogram was much higher than that of TNM stage. Time-dependent $\mathrm{C}$-index analysis also showed that our nomogram exhibited good prognostic accuracy in clinical outcome prediction for OTSCC patient when compared with TNM stage. At the same time, the decision curve analysis and calibration plots of 1-, 3- and 5-year OS showed that the nomogram had higher predictive accuracy and overall net benefit than TNM stage. Moreover, the nomogram could successfully classified OTSCC patients into high-risk and low-risk subgroups, and the high-risk patients had poor survival outcomes. Taken together, it seems that our nomogram could be helpful in predicting the OTSCC patient's outcome, and in treatment decisions-making for OTSCC patients. 

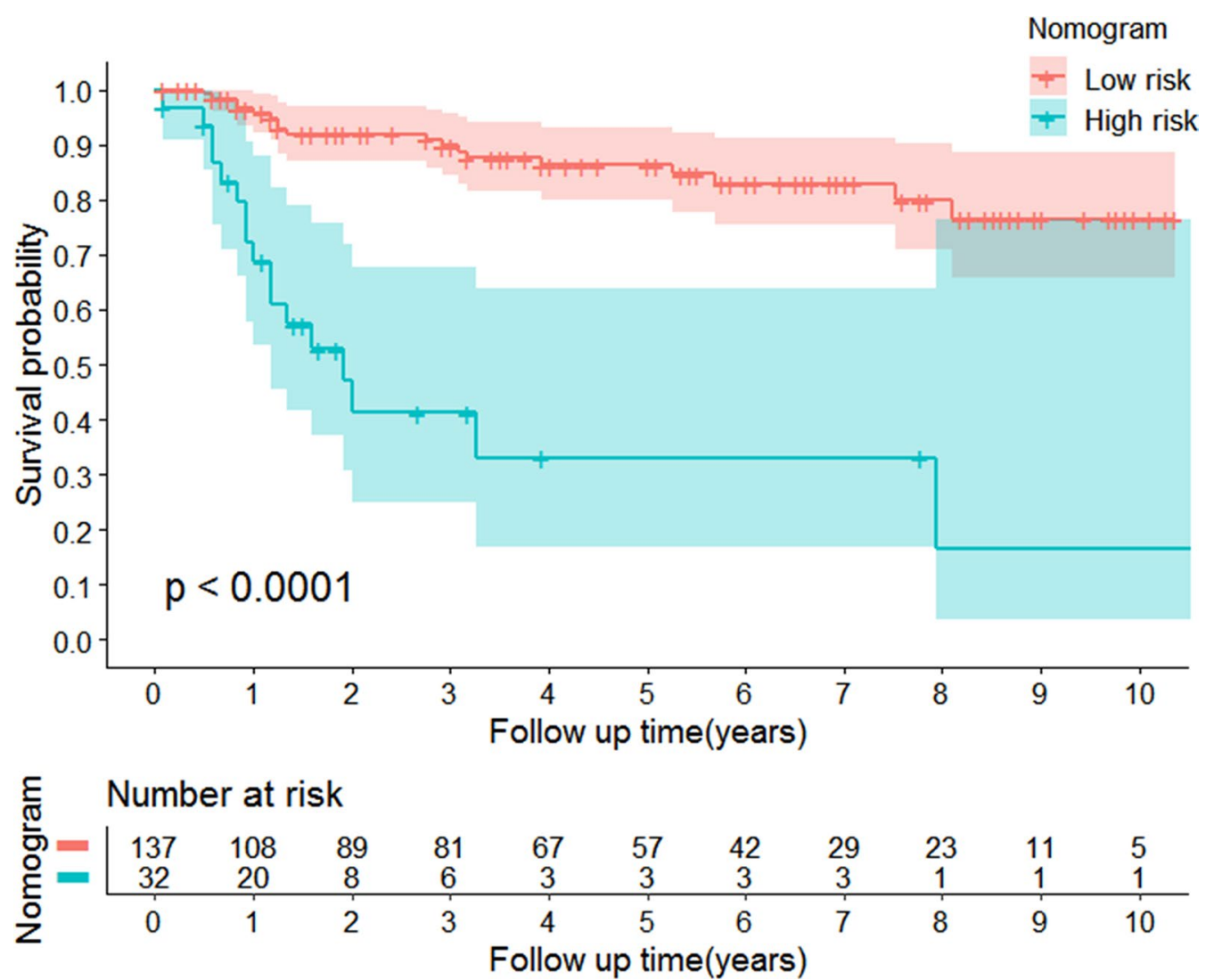

Fig. 7 Kaplan-Meier curve for OS based on the prediction of nomogram. Low risk, Total points < 195.13 for OS; High risk, Total points $\geq 195.13$ for OS. OS, overall survival

However, there are still some limitations in our study. First, our findings were based on a retrospective design, and thus, this study cannot exclude all potential bias. Second, our data was obtained from a single cancer center, and the sample size was small. A larger sample size from other institutions would be required to further validate our results. Finally, the endpoint of our study was OS, and more research on the disease-free survival should also be carried out in the future.

\section{Conclusions}

This study provided a novel nomogram based on clinical characteristics and serological inflammation markers with satisfactory performance when compared with traditional TNM stage system for individualized OS estimation. In the future, if a large-scale, multicenter prospective validation could be completed, our nomogram may be useful in clinical practice as a simple and readily available prognostic tool.

\begin{abstract}
Abbreviations
AIC: Akaike Information Criterion; AJCC: American Joint Cancer Committee; BF: B factor; BIC: Bayesian Information Criterion; C3: Complement 3; C4: Complement 4; C-index: Concordance index; CRP: C-reactive protein; HR: Hazard ratio; IgG: Immunoglobulin G; IgA: Immunoglobulin A; IgM: Immunoglobulin M; LMR: Lymphocyte-to-monocyte ratio; NLR: Neutrophil-to-lymphocyte ratio; OS: Overall survival; PLR: Platelet-to-lymphocyte ratio; TILs: Tumor-infiltrating lymphocytes; TNM: Tumor/node/metastasis; OTSCC: Oral tongue squamous cell carcinoma; WBC: White blood cell count; 95\% Cl: 95\% Confidence interval.
\end{abstract}

\section{Acknowledgements}

Not applicable.

\section{Authors' contributions}

YWL and WPK designed the study, analyzed and interpreted the data, and wrote the manuscript; BLH and ZHQ analyzed and interpreted the clinical data; LFW and BZ collected patient samples and clinical data, and analyzed the data; TYD and YL collected patient samples and clinical data; C-TL and LYC provided patient samples and clinical data; YHP, HPG and YWX conceptualized and designed the study, supervised the project, and revised the paper. All authors have approved the final version and agreed to publish the manuscript. All authors read and approved the final manuscript.

\section{Funding}

This work was supported by funding from the Natural Science Foundation of China [81972801 to Y-HP]; Guangdong Basic and Applied Basic Research Foundation [2018A030307079 to Y-HP, $2019 A 1515011873$ to Y-WX]; the Innovative and Strong School Project of Guangdong [2018KTSCX068 to Y-WX]; the 2020

Li Ka Shing Foundation Cross-Disciplinary Research Grant [2020LKSFG01B 
to Y-WX]; Guangdong Esophageal Cancer Institute Science and Technology Program [Q201906 to Y-WX] and Science and Technology Special Fund of Guangdong Province of China [190829105556145 to Y-WX].

\section{Availability of data and materials}

The datasets used and/or analysed during the current study are available from the corresponding author on reasonable request.

\section{Declarations}

\section{Ethics approval and consent to participate}

The authors confirm that the study has been approved by the Hospital Ethics Committee in Cancer Hospital of Shantou University Medical College and informed consents were obtained from all included participants. This study was performed in accordance with the ethical standards as laid down in the 1964 Declaration of Helsinki and its later amendments or comparable ethical standards.

\section{Consent for publication}

Not applicable.

\section{Competing interests}

The authors declare that they have no competing interests.

\begin{abstract}
Author details
${ }^{1}$ Department of Clinical Laboratory Medicine, The Cancer Hospital of Shantou University Medical College, Shantou 515041, Guangdong, People's Republic of China. ${ }^{2}$ Precision Medicine Research Center, Shantou University Medical College, Shantou 515041, Guangdong, People's Republic of China. ${ }^{3}$ Department of Otolaryngology, The Second Affiliated Hospital of Shantou University Medical College, Shantou 515041, Guangdong, People's Republic of China. ${ }^{4}$ Department of Head and Neck Surgery, The Cancer Hospital of Shantou University Medical College, Shantou 515041, Guangdong, People's Republic of China. ${ }^{5}$ Guangdong Esophageal Cancer Institute, The Cancer Hospital of Shantou University Medical College, Shantou 515041, Guangdong, People's Republic of China. ${ }^{6}$ Department of Otolaryngology-Head and Neck Surgery, The First Affiliated Hospital of Shantou University Medical College, Shantou 515041, Guangdong, People's Republic of China.
\end{abstract}

Received: 13 September 2021 Accepted: 14 December 2021 Published online: 27 December 2021

\section{References}

1. $\mathrm{Ng} \mathrm{JH}$, lyer NG, Tan MH, Edgren G. Changing epidemiology of oral squamous cell carcinoma of the tongue: a global study. Head Neck. 2017;39(2):297-304.

2. Li Y, Zhao Z, Liu X, Ju J, Chai J, Ni Q, et al. Nomograms to estimate longterm overall survival and tongue cancer-specific survival of patients with tongue squamous cell carcinoma. Cancer Med. 2017;6(5):1002-13.

3. Riemann M, Knipfer C, Rohde M, Adler W, Schuster M, Noeth E, et al. Oral squamous cell carcinoma of the tongue: prospective and objective speech evaluation of patients undergoing surgical therapy. Head Neck. 2016;38(7):993-1001.

4. Brenner H. Long-term survival rates of cancer patients achieved by the end of the 20th century: a period analysis. Lancet. 2002;360(9340):1131-5.

5. Shiboski $\mathrm{CH}$, Schmidt BL, Jordan RC. Tongue and tonsil carcinoma: increasing trends in the U.S. population ages 20-44 years. Cancer. 2005;103(9):1843-9.

6. Balachandran VP, Gonen M, Smith JJ, DeMatteo RP. Nomograms in oncology: more than meets the eye. Lancet Oncol. 2015;16(4):e173-80.

7. Iasonos A, Schrag D, Raj GV, Panageas KS. How to build and interpret a nomogram for cancer prognosis. J Clin Oncol. 2008;26(8):1364-70.

8. Gold JS, Gönen M, Gutiérrez A, Broto JM, García-del-Muro X, Smyrk $\mathrm{TC}$, et al. Development and validation of a prognostic nomogram for recurrence-free survival after complete surgical resection of localised primary gastrointestinal stromal tumour: a retrospective analysis. Lancet Oncol. 2009;10(11):1045-52.
9. Zhang JX, Song W, Chen ZH, Wei JH, Liao YJ, Lei J, et al. Prognostic and predictive value of a microRNA signature in stage II colon cancer: a microRNA expression analysis. Lancet Oncol. 2013;14(13):1295-306.

10. Kattan MW, Karpeh MS, Mazumdar M, Brennan MF. Postoperative nomogram for disease-specific survival after an R0 resection for gastric carcinoma. J Clin Oncol. 2003;21(19):3647-50.

11. Kattan MW, Gönen M, Jarnagin WR, DeMatteo R, D'Angelica M, Weiser M, et al. A nomogram for predicting disease-specific survival after hepatic resection for metastatic colorectal cancer. Ann Surg. 2008;247(2):282-7.

12. Zivanovic O, Jacks LM, lasonos A, Leitao MM Jr, Soslow RA, Veras E, et al. A nomogram to predict postresection 5-year overall survival for patients with uterine leiomyosarcoma. Cancer. 2012;118(3):660-9.

13. Yang $L$, Takimoto T, Fujimoto J. Prognostic model for predicting overall survival in children and adolescents with rhabdomyosarcoma. BMC Cancer. 2014;14:654.

14. Candido J, Hagemann T. Cancer-related inflammation. J Clin Immunol. 2013;33(Suppl 1):S79-84.

15. Nishijima TF, Muss HB, Shachar SS, Tamura K, Takamatsu Y. Prognostic value of lymphocyte-to-monocyte ratio in patients with solid tumors: a systematic review and meta-analysis. Cancer Treat Rev. 2015;41(10):971-8.

16. Li W, Tao L, Zhang L, Xiu D. Prognostic role of lymphocyte to monocyte ratio for patients with pancreatic cancer: a systematic review and metaanalysis. Onco Targets Ther. 2017;10:3391-7.

17. Tan D, Fu Y, Tong W, Li F. Prognostic significance of lymphocyte to monocyte ratio in colorectal cancer: a meta-analysis. Int J Surg. 2018;55:128-38.

18. Mao Y, Chen D, Duan S, Zhao Y, Wu C, Zhu F, et al. Prognostic impact of pretreatment lymphocyte-to-monocyte ratio in advanced epithelial cancers: a meta-analysis. Cancer Cell Int. 2018;18:201.

19. Sollie S, Santaolalla A, Michaud DS, Sarker D, Karagiannis SN, Josephs DH, et al. Serum immunoglobulin $\mathrm{G}$ is associated with decreased risk of pancreatic cancer in the Swedish AMORIS Study. Front Oncol. 2020;10:263.

20. Amin MB, Greene FL, Edge SB, Compton CC, Gershenwald JE, Brookland RK, et al. The eighth edition AJCC cancer staging manual: continuing to build a bridge from a population-based to a more "personalized" approach to cancer staging. CA Cancer J Clin. 2017;67(2):93-9.

21. Camp RL, Dolled-Filhart M, Rimm DL. X-tile: a new bio-informatics tool for biomarker assessment and outcome-based cut-point optimization. Clin Cancer Res. 2004;10(21):7252-9.

22. Lu Z, Yan W, Liang J, Yu M, Liu J, Hao J, et al. Nomogram based on systemic immune-inflammation index to predict survival of tongue cancer patients who underwent cervical dissection. Front Oncol. 2020;10:341.

23. Diakos Cl, Charles KA, McMillan DC, Clarke SJ. Cancer-related inflammation and treatment effectiveness. Lancet Oncol. 2014;15(11):e493-503.

24. Diem S, Schmid S, Krapf M, Flatz L, Born D, Jochum W, et al. Neutrophilto-Lymphocyte ratio (NLR) and Platelet-to-Lymphocyte ratio (PLR) as prognostic markers in patients with non-small cell lung cancer (NSCLC) treated with nivolumab. Lung Cancer. 2017;111:176-81.

25. Furukawa K, Kawasaki G, Naruse T, Umeda M. Prognostic significance of pretreatment lymphocyte-to-monocyte ratio in patients with tongue cancer. Anticancer Res. 2019;39(1):405-12.

26. Mahmoud SM, Paish EC, Powe DG, Macmillan RD, Grainge MJ, Lee AH, et al. Tumor-infiltrating CD8+ lymphocytes predict clinical outcome in breast cancer. J Clin Oncol. 2011;29(15):1949-55.

27. Gooden MJ, de Bock GH, Leffers N, Daemen T, Nijman HW. The prognostic influence of tumour-infiltrating lymphocytes in cancer: a systematic review with meta-analysis. Br J Cancer. 2011;105(1):93-103.

28. Ray-Coquard I, Cropet C, Van Glabbeke M, Sebban C, Le Cesne A, Judson I, et al. Lymphopenia as a prognostic factor for overall survival in advanced carcinomas, sarcomas, and lymphomas. Cancer Res. 2009;69(13):5383-91.

29. Schmidt H, Bastholt L, Geertsen P, Christensen IJ, Larsen S, Gehl J, et al. Elevated neutrophil and monocyte counts in peripheral blood are associated with poor survival in patients with metastatic melanoma: a prognostic model. Br J Cancer. 2005;93(3):273-8.

30. Lee YY, Choi CH, Sung CO, Do IG, Huh S, Song T, et al. Prognostic value of pre-treatment circulating monocyte count in patients with cervical cancer: comparison with SCC-Ag level. Gynecol Oncol. 2012;124(1):92-7.

31. Tsutsui S, Yasuda K, Suzuki K, Tahara K, Higashi H, Era S. Macrophage infiltration and its prognostic implications in breast cancer: the relationship with VEGF expression and microvessel density. Oncol Rep. 2005;14(2):425-31. 
32. Steidl C, Lee T, Shah SP, Farinha P, Han G, Nayar T, et al. Tumor-associated macrophages and survival in classic Hodgkin's lymphoma. N Engl J Med. 2010;362(10):875-85.

33. Lin S, Wang Y, Wang X, Yan B, Lou W, Di W. Serum immunoglobulin G $\mathrm{N}$-glycome: a potential biomarker in endometrial cancer. Ann Transl Med. 2020;8(12):748

34. Yi CH, Weng HL, Zhou FG, Fang M, Ji J, Cheng C, et al. Elevated corefucosylated lgG is a new marker for hepatitis B virus-related hepatocellular carcinoma. Oncoimmunology. 2015;4(12):e1011503.

35. Vučković F, Theodoratou E, Thaçi K, Timofeeva M, Vojta A, Štambuk J, et al. IgG glycome in colorectal cancer. Clin Cancer Res. 2016;22(12):3078-86.

36. Gudelj I, Lauc G, Pezer M. Immunoglobulin G glycosylation in aging and diseases. Cell Immunol. 2018;333:65-79.

37. Kessler A, Sollie S, Karagiannis SN, Walldius G, Hammar N, Van Hemelrijck M. Serum IgG is associated with risk of melanoma in the Swedish AMORIS study. Front Oncol. 2019;9:1095.

38. Sun W, Cheng M, Zhuang S, Chen H, Yang S, Qiu Z. Nomograms to predict survival of stage IV tongue squamous cell carcinoma after surgery. Medicine (Baltimore). 2019;98(26):e16206.

39. Saenthaveesuk P, Yang L, Zeng B, Xu M, Young S, Liao G, et al. Development and validation of multiparametric MRI-based nomogram for predicting occult metastasis risk in early tongue squamous cell carcinoma. BMC Cancer. 2021;21(1):408.

40. Mair M, Nair D, Nair S, Malik A, Mishra A, Kannan S, et al. Comparison of tumor volume, thickness, and T classification as predictors of outcomes in surgically treated squamous cell carcinoma of the oral tongue. Head Neck. 2018:40(8):1667-75.

41. Balasubramanian D, Subramaniam N, Missale F, Marchi F, Dokhe Y, Vijayan S, et al. Predictive nomograms for oral tongue squamous cell carcinoma applying the American Joint Committee on Cancer/Union Internationale Contre le Cancer 8th edition staging system. Head Neck. 2021;43(4):1043-55.

42. Huang $F, X u G, D u H$. A new nomogram for predicting overall survival and assisting postoperative adjuvant treatment decision-making in stage II oral tongue squamous cell carcinoma: a surveillance, epidemiology and end results (SEER) database analysis. J Oral Maxillofac Surg. 2021. https:// doi.org/10.1016/j.joms.2021.04.010.

43. Chang B, He W, Ouyang H, Peng J, Shen L, Wang A, et al. A prognostic nomogram incorporating depth of tumor invasion to predict long-term overall survival for tongue squamous cell carcinoma with R0 resection. $J$ Cancer. 2018;9(12):2107-15.

44. Mascitti M, Zhurakivska K, Togni L, Caponio VCA, Almangush A. Addition of the tumour-stroma ratio to the 8th edition American Joint Committee on Cancer staging system improves survival prediction for patients with oral tongue squamous cell carcinoma. Histopathology. 2020:77(5):810-22.

45. Jiang Q, Tang A, Long S, Qi Q, Song C, Xin Y, et al. Development and validation of a nomogram to predict the risk of occult cervical lymph node metastases in cNO squamous cell carcinoma of the tongue. Br J Oral Maxillofac Surg. 2019;57(10):1092-7.

46. Graupp M, Schaffer K, Wolf A, Vasicek S, Weiland T, Pondorfer P, et al. $C$-reactive protein is an independent prognostic marker in patients with tongue carcinoma - a retrospective study. Clin Otolaryngol. 2018. https:// doi.org/10.1111/coa.13102.

47. Abbate V, Dell'Aversana Orabona G, Salzano G, Bonavolontà P, Maglitto F, Romano A, et al. Pre-treatment Neutrophil-to-Lymphocyte Ratio as a predictor for occult cervical metastasis in early stage (T1-T2 cN0) squamous cell carcinoma of the oral tongue. Surg Oncol. 2018;27(3):503-7.

48. Wu CN, Chuang HC, Lin YT, Fang FM, Li SH, Chien CY. Prognosis of neutrophil-to-lymphocyte ratio in clinical early-stage tongue (CT1/T2N0) cancer. Onco Targets Ther. 2017;10:3917-24.

49. Shimazaki R, Takano S, Satoh M, Takada M, Miyahara Y, Sasaki K, et al. Complement factor $B$ regulates cellular senescence and is associated with poor prognosis in pancreatic cancer. Cell Oncol (Dordr). 2021. https://doi. org/10.1007/s13402-021-00614-z.

50. Peppas I, George G, Sollie S, Josephs DH, Hammar N, Walldius G, et al. Association of serum immunoglobulin levels with solid cancer: a systematic review and meta-analysis. Cancer Epidemiol Prev Biomark. 2020;29(3):527-38.

51. Schwartz-Albiez R, Monteiro RC, Rodriguez M, Binder CJ, Shoenfeld Y. Natural antibodies, intravenous immunoglobulin and their role in autoimmunity, cancer and inflammation. Clin Exp Immunol. 2009;158(Suppl 1):43-50.

52. Riihilä P, Nissinen L, Farshchian M, Kallajoki M, Kivisaari A, Meri S, et al. Complement component C3 and complement factor B promote growth of cutaneous squamous cell carcinoma. Am J Pathol. 2017;187(5):1186-97.

53. Kim SH, Lee MJ, Hwang HK, Lee $\mathrm{SH}$, Kim H, Paik YK, et al. Prognostic potential of the preoperative plasma complement factor $B$ in resected pancreatic cancer: a pilot study. Cancer Biomark. 2019;24(3):335-42.

54. Afshar-Kharghan V. The role of the complement system in cancer. J Clin Investig. 2017;127(3):780-9.

55. Ajona D, Ortiz-Espinosa S, Pio R. Complement anaphylatoxins C3a and C5a: emerging roles in cancer progression and treatment. Semin Cell Dev Biol. 2019;85:153-63.

56. Ling M, Murali M. Analysis of the Complement System in the Clinical Immunology Laboratory. Clin Lab Med. 2019;39(4):579-90.

\section{Publisher's Note}

Springer Nature remains neutral with regard to jurisdictional claims in published maps and institutional affiliations.
Ready to submit your research? Choose BMC and benefit from:

- fast, convenient online submission

- thorough peer review by experienced researchers in your field

- rapid publication on acceptance

- support for research data, including large and complex data types

- gold Open Access which fosters wider collaboration and increased citations

- maximum visibility for your research: over $100 \mathrm{M}$ website views per year

At BMC, research is always in progress.

Learn more biomedcentral.com/submissions 